\title{
Model for Random Hydrolysis and End Degradation of Linear Polysaccharides: Application to the Thermal Treatment of Mannan in Solution
}

\author{
Anders Nattorp, Martin Graf, Christian Spuhler, and Albert Renken* \\ Laboratoire de la Réaction Chimique et Electrochimique, E cole Polytechnique Fédérale de Lausanne, \\ 1015 Lausanne, Switzerland
}

\begin{abstract}
The kinetics for homogeneous hydrolysis of mannan is studied in a batch reactor at temperatures from 160 to $220^{\circ} \mathrm{C}$. A formate buffer ensures a pH of 3.8-4.0, measured at $25^{\circ} \mathrm{C}$. Samples are analyzed for ol igosaccharides up to a degree of polymerization of 6 and al so for the total amount of mannose after acid hydrolysis. A mathematical model with two reactions ( 1 , random hydrolysis of the glucosidic bonds; 2 , degradation of the reducing end of the molecule) describes accurately the time course of oligosaccharides. Optimized rate constants follow closely an Arrhenius relationship, with the degradation having a higher activation energy (140 kJ /mol) than the hydrolysis ( $113 \mathrm{~kJ} / \mathrm{mol}$ ). The mathematical model has the advantage that production of small molecules is independent of the initial chain-length distribution as long as the average initial chain length is some 5 times longer than the largest species measured. It can be applied to first-order depolymerization of other linear polymers with one link type in order to determine reaction rate constants or make predictions about molecular weight distribution on the base of known reaction rate constants.
\end{abstract}

\section{Introduction}

Hydrolysis of cellulose, amylose, and other linear polysaccharides is of importance in the paper, chemical, and food industries. It is likely that the homogeneous hydrolysis reaction pattern is the same for all of these compounds. Therefore, a mathematical model of homogeneous hydrolysis is of considerable interest, not only in the understanding the reactions of linear polysaccharides in solution but also as a basis for the devel opment of heterogeneous hydrolysis models necessary for solid materials.

Mannan is a linear polysaccharide suitable as a model compound for the development of a mathematical model of homogeneous hydrolysis. It serves as reserve polysaccharide in coffee beans ${ }^{1}$ and in palm fruits such as coconut, ${ }^{2}$ date, and ivory nut. ${ }^{3}$ It is also present in certain algae. ${ }^{4}$ Mannan has a backbone of $\beta$ - $(1,4)$-linked mannose units with short branches consisting of one $\beta$-(1,6)-linked galactose unit, which represent less than $5 \%$ of the total mass. It resembles cellulose in many respects. They both have $\beta-(1,4)$ linking, leading to intramolecular bonds that favor a straight conformation of chains. The straight inflexible chains can form a compact supermolecular structure with many intermolecular hydrogen bonds. Such a supermolecular structure is often crystalline and difficult to render soluble. Also, observed precipitation of unbranched $\beta$-(1,4)-linked polysaccharides is an indication of the stability of the structure. ${ }^{5}$ The degree of polymerization (DP) of mannan $(50-200)^{3}$ varies with its origin but is al ways lower than that of cellulose (300-3000). ${ }^{6}$ This is one reason mannan crystals can be more easily dissolved than those of cellulose, for which either strong electrolytes (such as concentrated acids) ${ }^{7}$ or extreme temperatures (370

* To whom correspondence should be addressed. E-mail: al bert.renken@epfl.ch. Fax: +41-21-693 3190. $\left.{ }^{\circ} \mathrm{C}\right)^{8}$ are required. Consequently, mannan is suitable for evaluation of a homogeneous model, whereas cellulose is not.

The depolymerization of polysaccharides by hydrolysis proceeds by protonation of the glucosidic oxygen ( $\mathrm{F}$ igure $1)$. This step is reversible and fast. The rate-limiting step is most likely the breaking of the bond which forms a carbocation with an energetically unfavorable conformation. The carbocation is then quickly stabilized by the free electron pairs of a water molecule, and so the new oligo- or polysaccharide end is formed. ${ }^{9}$ Thus, the rate should be proportional to the concentration of protonated bonds and consequently to the proton concentration, although some evidence exists that this is not the case for a thermal hydrolysis. ${ }^{10}$ In this study, the proton concentration is kept constant during trials and the apparent hydrolysis rate constant is determined.

Thermal hydrolysis occurs at temperatures above 150 ${ }^{\circ} \mathrm{C}$ without addition of strong acids. Under these conditions significant degradation al so takes place. In polysaccharides the monosaccharide unit at one end is expected to be much more susceptible to degradation than the others. In fact, monosaccharides in solution exist as a mix of the aldehyde form and ring structures, hemiacetals, formed by condensation of the carbonyl group with a hydroxyl group at the far end of the chain (Figure 2). ${ }^{11}$ At normal temperatures this ring form is more abundant than the open-chain form. It is also more resistant to degradation; actually it has been shown that the degradation rate of a monosaccharide is strongly dependent on the fraction of the open-chain form. ${ }^{12}$ In polysaccharides the monosaccharide units are blocked in the ring form. Only the monosaccharide situated at the end of the chain can undergo ring opening. This end is called the reducing end because of the accessible aldehyde function. The high reactivity of the reducing end was observed experimentally for cellobiose. ${ }^{13}$ 


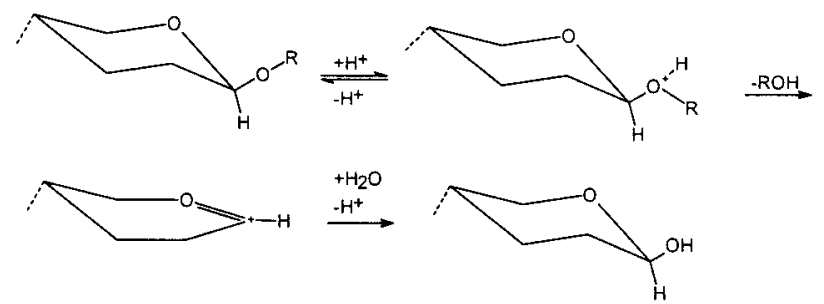

Figure 1. Mechanism of hydrolysis of a glucosidic bond. ${ }^{9}$
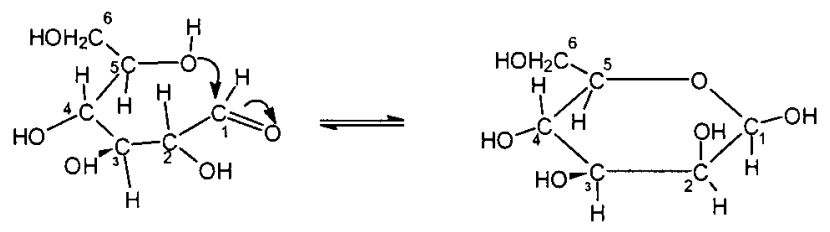

Figure 2. Ring closing: formation of a $\beta$-glucopyranose (mannose).

Previous modeling of homogeneous hydrolysis includes, on the one hand, the development of rate equations for thermal hydrolysis of ol igosaccharides ${ }^{14,15}$ and, on the other hand, the determination of acid hydrolysis rates from modeling of experiments with a polysaccharide, dextran. ${ }^{16}$ In addition, Monte Carlo simulation has been performed to predict glucose production from amylose. 17

However, this is to our knowledge the first modeling of thermal homogeneous hydrolysis of polysaccharides. Hydrolysis kinetics of mannan in solution is investigated at four different temperatures between 160 and $220^{\circ} \mathrm{C}(6-23$ bar). The concentrations of the mono- and oligosaccharides from DP 1 to 6 as well as the total mannose of all of the samples are analyzed with HPLC. The time course of the concentrations for the different degrees of polymerization reflects the hydrolysis of mannan.

\section{Experimental Section}

Buffered mannan solutions were heated in a batch reactor. Samples were withdrawn at chosen intervals and analyzed with HPLC. A comparative trial with another starting material, mannose, was performed with the same procedure. The experimental and analytical procedure is described below.

Reactor and Procedure. A $2 \mathrm{~L}$ batch reactor with a propeller stirrer (diameter $50 \mathrm{~mm}$; speed $10 \mathrm{rps}$ ) was used (Figure 3). The mannan solution was heated with steam in the jacket until the temperature was $50{ }^{\circ} \mathrm{C}$ below the reaction temperature, and then steam was injected into the sol ution through a stoppered tube with four radial exits $(1 \times 15 \mathrm{~mm})$ for the last $50^{\circ} \mathrm{C}$. The reaction time was counted from the end of the steam injection, which lasted about $5 \mathrm{~s}$. The temperature was measured by a thermocouple in the solution and controlled with steam in the jacket with a precision of \pm 1 ${ }^{\circ} \mathrm{C}$. Samples were withdrawn into a tube, in which they were cooled to $50{ }^{\circ} \mathrm{C}$ within $30 \mathrm{~s}$. Thus, the reaction time during heating and cooling was small compared to the duration of the trial (1 min or more).

Mannan (Megazyme Inc., Bray, I reland) was dissolved in water ( $1 \mathrm{~g}$ in $1 \mathrm{~L}$ ) by heating at $200{ }^{\circ} \mathrm{C}$ for $15 \mathrm{~s}$ at neutral $\mathrm{pH}$. We observed that $23 \%$ of the mannan was degraded during the solubilization. However, this is taken into account because the modeling was based on the mannan concentration in the initial sample. The average degree of polymerization probably also de-

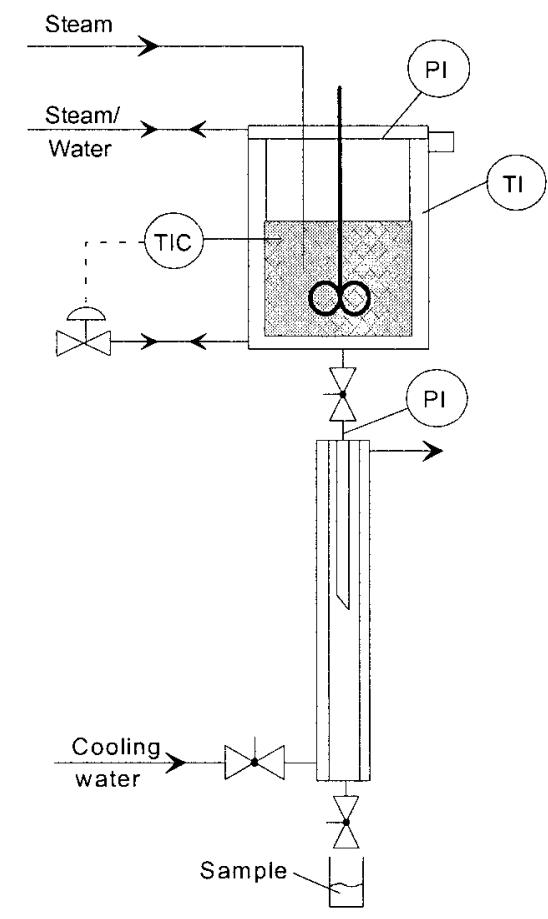

Figure 3. Experimental setup.

creased, but it remained well above the limit of detection (DP 6) because the initial amount of oligosaccharides was low (Figure 4). Consequently, the degradation in the solubilization step did not influence the results of the modeling. After cooling a clear solution was obtained, to which a formate buffer (see below) was added, and the trial was started.

Buffer. As mentioned before, the hydrolysis is probably influenced by the proton concentration. Therefore, the solutions in this study were buffered. During the solubilization step a neutral $\mathrm{pH}$ was ensured by a phosphate buffer $\left(2.5 \mathrm{mmol} / \mathrm{L} \quad \mathrm{NaH}_{2} \mathrm{PO}_{4} / 2.5 \mathrm{mmol} / \mathrm{L}\right.$ $\left.\mathrm{Na}_{2} \mathrm{HPO}_{4}\right)$. Then, a formate buffer $(10 \mathrm{mmol} / \mathrm{L} \mathrm{HCOOH} /$ $10 \mathrm{mmol} / \mathrm{L} \mathrm{NaCOOH}$ ) was added before the trial. The resulting $\mathrm{pH}$ was 3.9, so the phosphate could be considered to be quantitatively present as the conjugate acid, and the mix consequently behaved as an unbalanced formate buffer $(7.5 \mathrm{mmol} / \mathrm{L} \mathrm{HCOOH} / 12.5 \mathrm{mmol} / \mathrm{L}$ $\mathrm{NaCOOH}$ ).

The stability of the formic buffer was tested without mannan. Its $\mathrm{pH}$ remained unchanged after heating to $220{ }^{\circ} \mathrm{C}$ for $30 \mathrm{~min}$.

The $\mathrm{pH}$ measured in samples at $25^{\circ} \mathrm{C}$ decreased slightly with time, probably because of the formation of organic acids during the degradation of mannan. It passed from 3.9-4.0 in the initial samples to 3.8-3.9 in the final ones. With these data the proton concentration at high temperature can be calculated. ${ }^{18}$ This is important information, but it will not be used to calculate real hydrolysis rate constants from the apparent ones, because their dependence on the proton concentration is unknown.

HPLC. The oligomannan and total mannose content of samples were analyzed on a DIONEX system.

A Carbopac PA-100 column and precolumn (DIONEX) were operated with a flow of $1 \mathrm{~mL} / \mathrm{min}$, and the effluent was mixed with $0.3 \mathrm{~mL} / \mathrm{min}$ of a $0.3 \mathrm{~mol} / \mathrm{L} \mathrm{NaOH}$ solution before entering the pulsed amperometry detector. The response factors were determined with standard solutions of mannose (>99\%, Fluka, Hauppauge, NY), mannobiose, mannotetraose, and mannohexaose 

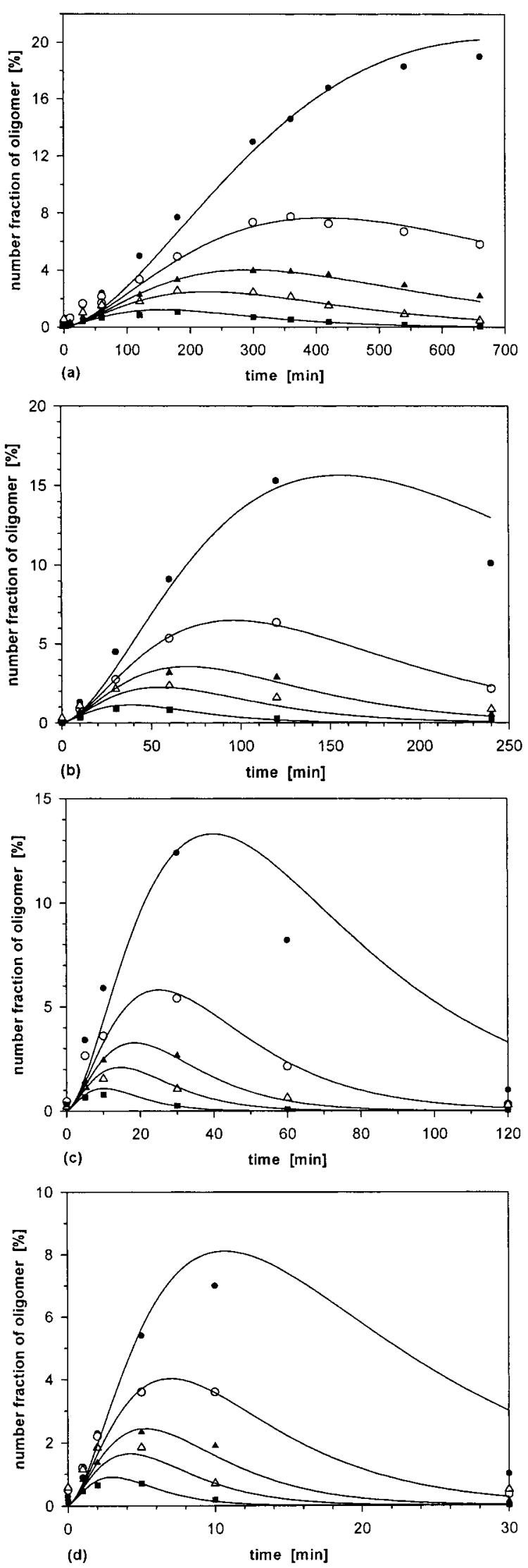

Figure 4. Comparison of experimental data with calculated curves. $\mathrm{T}=160$ (a), 180 (b), 200 (c), and $220^{\circ} \mathrm{C}$ (d). Symbols: solid circle, mannose; empty circle, mannobiose; solid triangle, mannotriose; empty triangle, mannotetraose; solid square, mannohexaose. Lines show the model prediction.

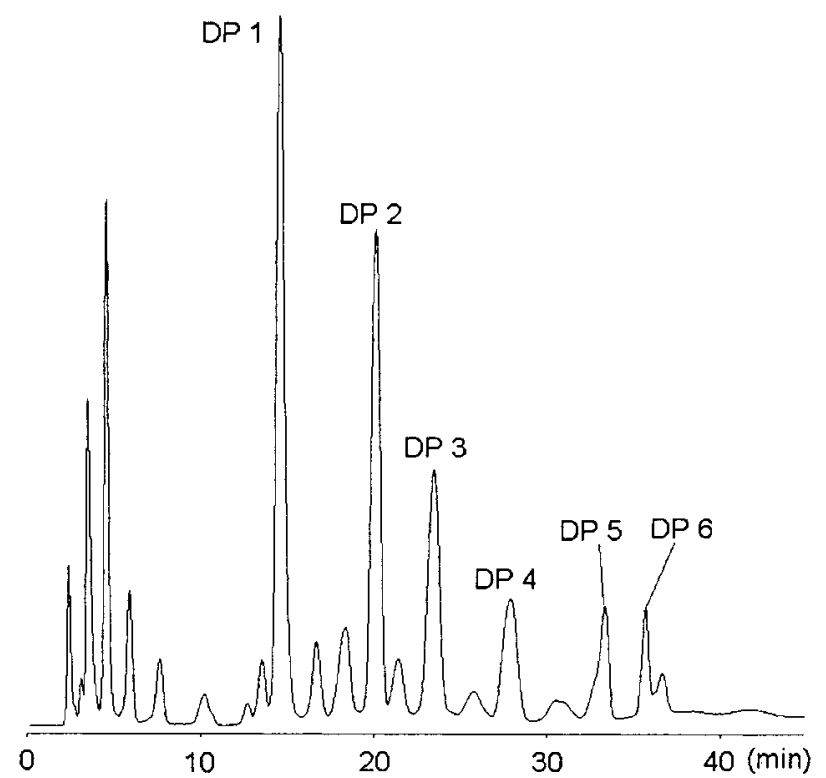

Figure 5. Example of a chromatogram for HPLC separation of oligomannan. Two linear $\mathrm{NaOH}$ elution gradients were used: 1.67 $(\mathrm{mmol} / \mathrm{L}) / \mathrm{min}$ for the first $15 \mathrm{~min}$ (starting with $0 \mathrm{mmol} / \mathrm{L}$ ) and then $1.11(\mathrm{mmol} / \mathrm{L}) / \mathrm{min}$.

(all Megazyme). The total mannose content of the oligomers was analyzed by acid hydrolysis (see below) and comparison to the mannose standard in order to take impurities (10-15\%) into account. Two different gradients were used to elute the different compounds. On the one hand, different monosaccharides (arabinose, galactose, glucose, and mannose) were best separated using only water. On the other hand, mannose and ol igomannans up to a degree of polymerization of 6 were eluted using a gradient with increasing $\mathrm{NaOH}$ concentration, allowing a good separation of peaks (Figure 5). Prior to analysis of oligosaccharides, the samples were filtered on C18 cartridges (Supel co, Bell efonte, PA), to remove nonpol ar compounds and particles. For analysis of the total mannose, the samples were hydrolyzed for $2 \mathrm{~h}$ at $100{ }^{\circ} \mathrm{C}$ in $7 \% \mathrm{H}_{2} \mathrm{SO}_{4}$ and then filtered through C18 cartridges.

The standard deviation for the whole process (heat treatment and analysis) was $7 \%$ relative, based on oligomer concentrations after $60 \mathrm{~min}$ at $160{ }^{\circ} \mathrm{C}$ in two identical trials.

\section{Results}

The peaks in the chromatograms (Figure 5) corresponding to mannose and ol igomannans of DP 2-6 were identified by comparison with a standard solution. The peak of mannopentaose on the chromatogram is exceptionally high for all samples. It is probable that during hydrolysis another substance is formed, which has the same retention time as mannopentaose. If the two signals overlap, the high peak for mannopentaose can be explained. Because it does not fit the pattern observed for the other ol igosaccharides, mannopentaose is disregarded.

The concentration courses of the ol igomers are shown in Figure 4 for different temperatures, together with the model prediction. All species pass through a maximum, with the hexamer maximum reached first and that of the monomer reached last. This is consistent with a depolymerization, an evolution toward smaller species. Because the monomer also decreases with time, there 


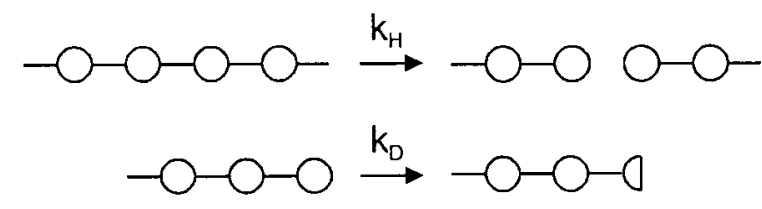

Figure 6. Schematic representation of (a) hydrolysis of a glycosidic bond and (b) degradation of the terminal unit at the reducing end.

must be a degradation reaction consuming this species. The maximum number fraction for the monomer is dependent on temperature. It decreases from $17 \%$ at 160 ${ }^{\circ} \mathrm{C}$ to $7 \%$ at $220^{\circ} \mathrm{C}$, indicating that the degradation reaction becomes increasingly important with temperature.

Model Formulation. Depolymerization of chain molecules through scission of the chemical bonds that link two monomer units is a problem frequently encountered in polymer science. Practical examples include weathering, biodegradability, and recycling of plastics. ${ }^{19}$ The cleavage of bonds can be due to shear action, ${ }^{20,21}$ irradiation, 22,23 or chemical attack;24 here, we are concerned with the special case of hydrolysis in polysaccharides. ${ }^{16}$

Three distinct methodologies are used to model the kinetics of depolymerization reactions. First, the fragmentation process can be treated as a statistical problem, using mathematical techniques such as combinatorial analysis ${ }^{25}$ or Markov chain theory. ${ }^{26}$ Second, the stochasticity of the process can be handled by performing simulations, e.g. using Monte Carlo methods, to compute the evolution of the reacting mass. ${ }^{17,27}$ Third, a deterministic approach where the kinetic mechanism is expressed and population-balance equations are expli citly solved can al so be applied. In this latter case, the chain length can be treated either as a continuous ${ }^{28}$ or as a discrete variable; an example of discrete treatment is the work by Basedow et al., ${ }^{16}$ who devel oped a set of equations describing the evolution of the chainlength distribution (CLD) and obtained an analytical solution for the special case of random scission.

In this work, we develop a kinetic model based on the determi nistic approach, for the case of random scission of the bonds linking the repeating units and simultaneous degradation of the terminal unit of the chains, to account for the hydrolysis of polysaccharides in solution.

Mannan undergoes depolymerization through acidcatalyzed hydrolysis of the $\beta$ - $(1,4)$-glycosidic bonds linking the monosaccharide units to each other. The ultimate state of the hydrolysis process should consist of all of the polymer being fully converted to mannose (the monomer). Because it is experimentally observed that the mannose concentration decreases after long reaction times, a further possibility of reaction has to be introduced. This is done by all owing the reducing end of the polymer chains to undergo degradation (Figure 6).

Evidence for a degradation reaction is supported by a recent work on the decomposition of cellobiose (cellulose dimer) in sub- and supercritical water. ${ }^{13}$ In the reaction pathway that the authors were able to elucidate, pyrolysis occurs at the reducing end, leading to glucosyl erythrose or glucosyl glycolaldehyde as degraded species. Because the chemical structures of mannan and cellulose are similar, this degradation reaction is incorporated in the overall depolymerization reaction scheme of mannan, consequently written as follows:

$$
\begin{aligned}
& P_{i} \stackrel{k_{H}}{\longrightarrow} P_{j}+P_{i-j} \quad i=2,3, \ldots \quad j=1,2, \ldots, i-1 \\
& P_{i} \stackrel{k_{D}}{\longrightarrow} D_{i} \quad i=1,2, \ldots \\
& D_{i} \stackrel{k_{H}}{\longrightarrow} P_{j}+D_{i-j} \quad i=2,3, \ldots \quad j=1,2, \ldots, i-1
\end{aligned}
$$

where $P_{i}$ stands for a mannan chain of length $i$ (consisting of $i$ monosaccharide units) and $D_{i}$ for a chain of length $\mathrm{i}$ with a degraded terminal monosaccharide unit. Reaction 1 represents the hydrolysis of a nondegraded chain, reaction 2 degradation at the reducing end, and reaction 3 hydrolysis of a degraded chain. The same hydrolysis rate constant $\mathrm{k}_{\mathrm{H}}$ is assigned to reactions 1 and 3 in accordance with Kabyemela's observation that cellobiose, glucosyl erythrose, and glucosyl glycolaldehyde undergo hydrolysis at the same rate.

Hydrolysis and degradation are assumed to be both irreversible, first-order processes (at constant pH). Furthermore, $\mathrm{k}_{\mathrm{H}}$ is assumed to be independent of the chain length and of the bond position in the chain, so that scission occurs at random. Final ly, the degradation rate constant $k_{D}$ is also taken as independent of the chain length.

The state of the reacting mass at time $t$ is described by the following two vectors, representing the CLDs of the two populations,

$$
\vec{P}(t)=\left(\begin{array}{l}
P_{1}(t) \\
P_{2}(t) \\
\vdots \\
P_{i}(t) \\
\vdots \\
P_{r}(t)
\end{array}\right) \quad \text { and } \quad \vec{D}(t)=\left(\begin{array}{l}
D_{1}(t) \\
D_{2}(t) \\
\vdots \\
D_{i}(t) \\
\vdots \\
D_{r}(t)
\end{array}\right)
$$

where $P_{i}(t)$ and $D_{i}(t)$ stand for the number of nondegraded and degraded chains of length $\mathrm{i}$ at time $\mathrm{t}$, respectively, and $r$ is the highest chain length under consideration.

To obtain the time evolution of the CLDs, the material balance of $P_{i}$ and $D_{i}$ must be expressed. This includes several contributions. First, hydrolysis of any of the i -1 bonds of a chain of length i leads to its destruction:

$$
\begin{aligned}
\left(\frac{d P_{i}}{d t}\right)_{1}=-k_{H}(i-1) P_{i} \quad \text { and } \\
\left(\frac{d D_{i}}{d t}\right)_{1}=-k_{H}(i-1) D_{i}
\end{aligned}
$$

Second, chains of length i can also be created by hydrolysis of higher chains. Because a $\mathrm{P}_{i}$ molecule can be split off from a $P_{j}$ molecule $(j>i)$ by scission of either its ith or its $(j-i)$ th bond, this contribution is expressed as

$$
\left(\frac{d P_{i}}{d t}\right)_{2}=2 k_{H} \sum_{j=i+1}^{r} P_{j}
$$

This expression remains valid for the special case $\mathrm{j}=$ $2 i$, because the fact that there is only one suitable bond (the central one) in this case is compensated by the fact that scission of this bond leads to the formation of two $\mathrm{P}_{\mathrm{i}}$ molecules.

Third, the corresponding expressions for the hydrolysis of $D_{j}$ chains are similarly written as 


$$
\left(\frac{d P_{i}}{d t}\right)_{3}=\left(\frac{d D_{i}}{d t}\right)_{3}=k_{H} \sum_{j=i+1}^{r} D_{j}
$$

Finally, the effect of degradation at the reducing end is taken into account:

$$
\left(\frac{d P_{i}}{d t}\right)_{4}=-k_{D} P_{i} \quad \text { and } \quad\left(\frac{d D_{i}}{d t}\right)_{4}=k_{D} P_{i}
$$

When the Damköhler number $\mathrm{Da}=\mathrm{k}_{\mathrm{H}} \mathrm{t}$ is introduced for hydrolysis in a batch reactor and $\beta=\mathrm{k}_{\mathrm{D}} / \mathrm{k}_{\mathrm{H}}$ is defined as the ratio of the degradation rate constant to the hydrolysis rate constant, the above kinetic contributions are put into the material balances to yield the following pair of coupled differential vectorial equations:

$$
\begin{aligned}
& \frac{d \vec{P}}{d D a}=\mathbf{A} \vec{P}+\mathbf{B} \vec{D} \\
& \frac{d \vec{D}}{d D a}=\beta \mathbf{I P}+\mathbf{C} \vec{D}
\end{aligned}
$$

where $\mathbf{A}, \mathbf{B}$, and $\mathbf{C}$ are the following $r \times r$ square matrices (I is the unit matrix):

$$
\begin{aligned}
& \mathbf{A}=\left[\begin{array}{lllll}
-\beta & 2 & 2 & \cdots & 2 \\
0 & -(\beta+1) & 2 & \cdots & 2 \\
0 & 0 & -(\beta+2) & \cdots & 2 \\
\vdots & \vdots & \vdots & \ddots & \vdots \\
0 & 0 & 0 & \cdots & -(\beta+r-1)
\end{array}\right] \\
& \mathbf{B}=\left[\begin{array}{lllll}
0 & 1 & 1 & \cdots & 1 \\
0 & 0 & 1 & \cdots & 1 \\
0 & 0 & 0 & \cdots & 1 \\
\vdots & \vdots & \vdots & \ddots & \vdots \\
0 & 0 & 0 & \cdots & 0
\end{array}\right] \\
& \mathbf{C}=\left[\begin{array}{lllll}
0 & 1 & 1 & \cdots & 1 \\
0 & -1 & 1 & \ddots & 1 \\
0 & 0 & -2 & \cdots & 1 \\
\vdots & \vdots & \vdots & \ddots & \vdots \\
0 & 0 & 0 & \cdots & -(r-1)
\end{array}\right]
\end{aligned}
$$

Equations 9 and 10 are numerically solved using Gear's BDF-DIFSUB algorithm ${ }^{29}$ in the SimuSolv integration package, ${ }^{30}$ to give the ti me course of the CLDs. The prediction for the first six oligomers (mannose $\mathrm{P}_{1}$ to mannohexaose $\mathrm{P}_{6}$ ) is represented in Figure 7 , for $\beta$ $=2$ and a starting monodisperse distribution of mannan chains of DP 50. The time profiles are given as the number fraction of the considered oligomer as a function of the Damköhler number, with the number fraction defined as the ratio of the number of molecules of the considered species to the total number of repeating units in the system; this definition is chosen to better separate the curves for the different oligomers on the graphs:

$$
\begin{aligned}
& \text { number fraction } P_{i}=\frac{P_{i}}{\sum_{i}\left(i P_{i}+i D_{i}\right)} \\
& \text { number fraction } D_{i}=\frac{D_{i}}{\sum_{i}\left(i P_{i}+i D_{i}\right)}
\end{aligned}
$$

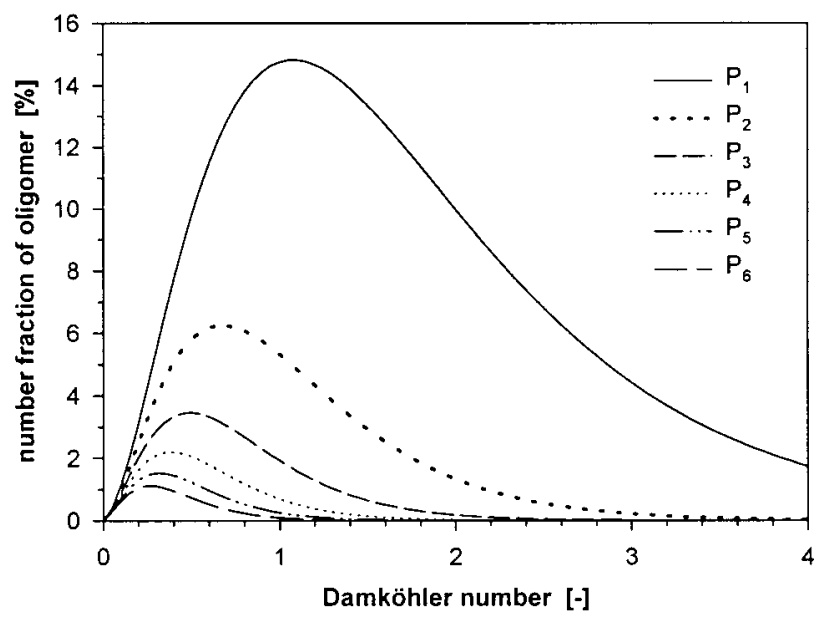

Figure 7. Model prediction for the oligomers $\mathrm{P}_{1}-\mathrm{P}_{6} ; \beta=2$, initial monodisperse CLD at $\mathrm{DP}_{0} 50$.

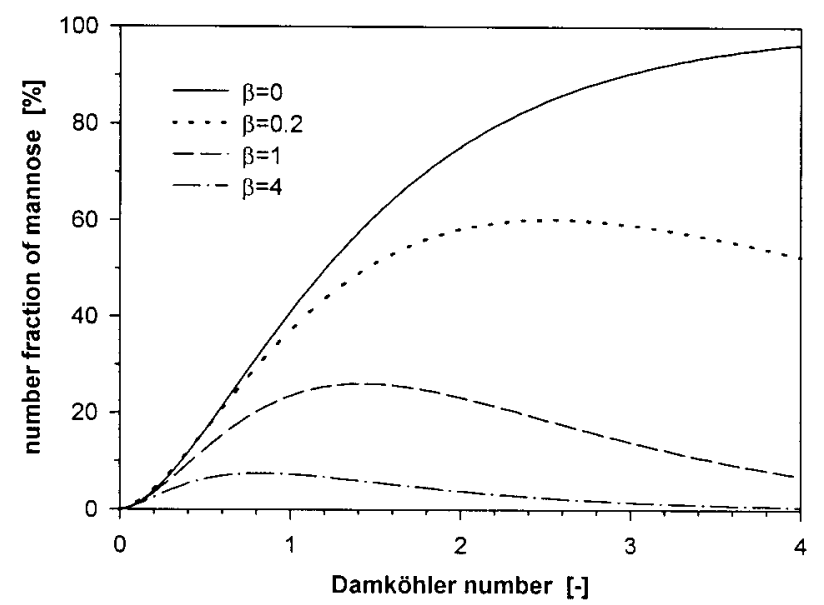

Figure 8. Effect of degradation on mannose $\left(\mathrm{P}_{1}\right)$ production; model prediction for $\beta=\mathrm{k}_{\mathrm{D}} / \mathrm{k}_{\mathrm{H}}=0,0.2,1$, and 4 ; initial monodisperse CLD at $\mathrm{DP}_{0} 50$.

Figure 7 shows that the $\mathrm{P}_{\mathrm{i}}$ oligomers are transient species of the depolymerization process, with a maximum concentration, which is logically positioned earlier for higher oligomers. Also, the problem of choosing an appropriate reaction time in order to achieve optimal selectivity is clearly apparent.

The effect of degradation is investigated by solving the dynamic model for four values of $\beta$. Again, the initial condition is a monodisperse CLD at DP $=50$. The time course of the monomer $\mathrm{P}_{1}$ is given in Figure 8 . F or $\beta=$ 0 (no degradation), the number fraction of mannose tends logically toward 1, because at complete conversion of the glycosidic linkages, the final state of hydrolysis consists of pure mannose. When degradation is allowed to take place $(\beta=0.2,1$, and 4$)$, the mannose concentration is reduced and tends toward zero at infinite time, with the final state of the system consisting of pure degraded monomer $D_{1}$. The reduction in mannose content is more pronounced with increasing values of $\beta$.

Next, the sensitivity of the model to the initial distribution of mannan molecules in the reactor is checked. F or this purpose, simulations were performed starting with four different monodisperse CLDs of DP $10,25,50$, and 75 . Figure 9 shows the time course of mannohexaose $\mathrm{P}_{6}$ for these different cases with $\beta=2$ : whereas initial DP values rising from 10 to 50 still influence the mannohexaose profile, the effect of a further increase to DP 75 is barely noticeable, certainly 


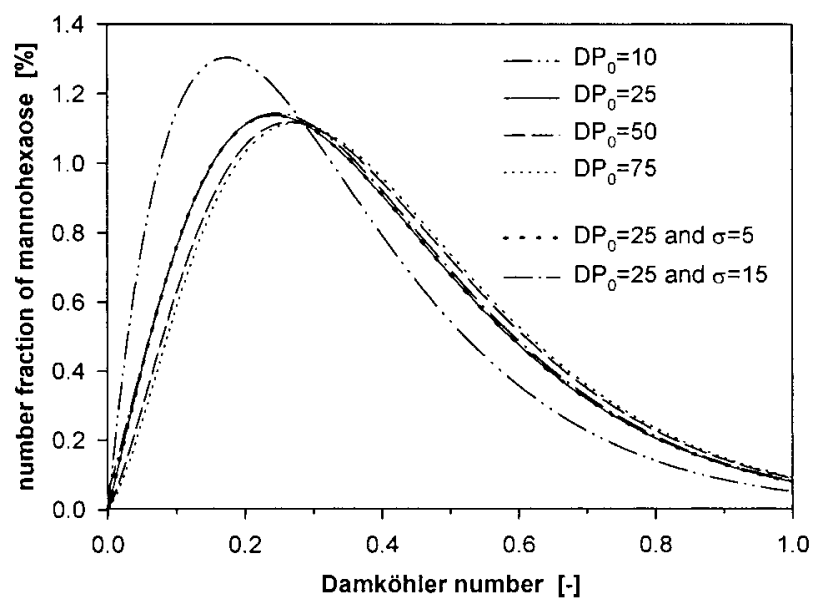

Figure 9. Model sensitivity to the initial distribution of mannan molecules; initial monodisperse CLDs ( $\mathrm{DP}_{0} 10,25,50$, and 75); initial Gaussian CLDs at average DPo $25(\sigma=5$ and 15); mannohexaose $\mathrm{P}_{6}, \beta=\mathrm{k}_{\mathrm{D}} / \mathrm{k}_{\mathrm{H}}=2$.

within experimental error. Moreover, simulations were al so carried out starting with Gaussian CLDs of different standard deviations $\sigma=5$ and 15 around the number-average value of DP 25 . The result is also shown in Figure 9 for mannohexaose $\mathrm{P}_{6}$ and $\beta=2$. Clearly, there is practically no effect of the dispersion of the initial CLD.

An important consequence of these results is that the developed model can be safely used to extract kinetic parameters from experimental oligomer data even if the initial distribution of mannan chains in solution is not exactly known, provided the initial number-average chain length is high enough.

Finally, a comparison of the relative amounts of the two populations is represented in Figure 10, where the number fraction of mannose units in the nondegraded oligomers $\left(P_{1}\right.$ to $\left.P_{6}\right)$ is shown together with the number fraction of mannose units (i.e., the number fraction of nondegraded repeating units) in the degraded oligomers $\left(D_{1}\right.$ to $D_{6}$ ), for $\beta=2$. At this value of $\beta$ (typical for our experiments), the maximum mannose content of $\mathrm{P}$ oligomers is about twice the maximum mannose content of $\mathrm{D}$ oligomers.

\section{Performance of the Model and Discussion}

The $\mathrm{D}$ species in the model have not been identified. However, there are peaks in the chromatogram that might correspond to those species (Figure 5). The peaks between DP 1 and 2, the one between DP 2 and 3, and the one between DP 3 and 4 all show trends similar to those of DP 2-4, i.e., a maximum at approximately the same reaction time. The ratio of the area of such an unidentified peak to the area of the next identified peak in the chromatogram is between 0.2 and 1 . It is possible that these unidentified peaks correspond to the $D$ species.

The parameters $k_{H}$ and $k_{D}$ in the mathematical model are adapted to fit simultaneously the concentration courses for DP 1-4 and 6 (Figure 4). The optimization is made either with a Nelder-Mead search method or with a generalized reduced gradient method, both features of the SimuSolv integration package. ${ }^{30}$ The model fits experimental data well, and no particular area where the model fails has been observed. The rate constants (Table 1) follow precisely an Arrhenius relationship (Figure 11). The activation energy for degrada-

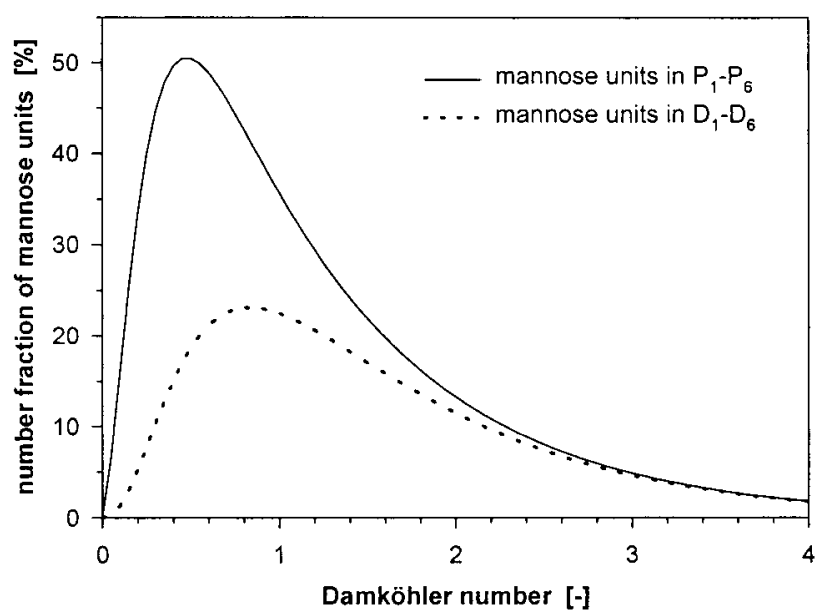

Figure 10. Comparison of the two populations of oligomers, for a value of $\beta=\mathrm{k}_{\mathrm{D}} / \mathrm{k}_{\mathrm{H}}=2$ typical for our experiments; initial monodisperse CLD at $\mathrm{DP}_{0} 50$.

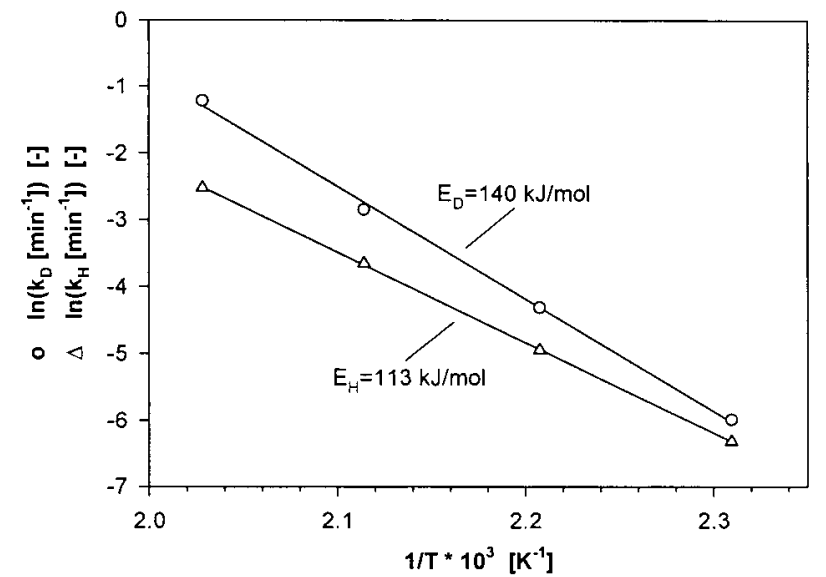

Figure 11. Influence of the temperature on the fitted rate constants.

Table 1. Reaction Rate Constants for Hydrolysis and Degradation at Different Temperatures

\begin{tabular}{ccc}
\hline $\operatorname{temp}\left({ }^{\circ} \mathrm{C}\right)$ & $\mathrm{k}_{\mathrm{H}}\left(\mathrm{min}^{-1}\right)$ & $\mathrm{k}_{\mathrm{D}}\left(\mathrm{min}^{-1}\right)$ \\
\hline 160 & 0.0018 & 0.0025 \\
180 & 0.0071 & 0.0134 \\
200 & 0.0260 & 0.0580 \\
220 & 0.0800 & 0.2960
\end{tabular}

tion, $140 \mathrm{~kJ} / \mathrm{mol}$, is higher than that for hydrolysis, 113 $\mathrm{kJ} / \mathrm{mol}$. This leads, as expected, to a $\mathrm{k}_{\mathrm{D}} / \mathrm{k}_{\mathrm{H}}$ ratio increasing with temperature. The total mannose content of the samples is not used as a base for optimization but as a comparison. The model with the rate constants determined on the basis of oligomer measurements agrees well with the experimental data of the total mannose for the different reaction temperatures (Figure 12).

No literature values for the rate of mannan depolymerization exist, but similar studies have been made on cellobiose. In unbuffered trials between 300 and 400 ${ }^{\circ} \mathrm{C}, 13$ the activation energy for cellobiose hydrolysis was $109 \mathrm{~kJ} / \mathrm{mol}$, which is close to the value in the present paper, but in another study at temperatures between 180 and $250{ }^{\circ} \mathrm{C}, 10$ a considerably higher activation energy was found, $136 \mathrm{~kJ} / \mathrm{mol}$. When degradation of glucose was studied under the same conditions, the activation energy was some $15 \mathrm{~kJ} / \mathrm{mol}$ lower than that of the hydrolysis, 96 and $121 \mathrm{~kJ} / \mathrm{mol}$, respectively. ${ }^{31,32}$ These research groups thus observed a $\mathrm{k}_{\mathrm{D}} / \mathrm{k}_{\mathrm{H}}$ ratio decreasing with temperature. This is in contrast to the 


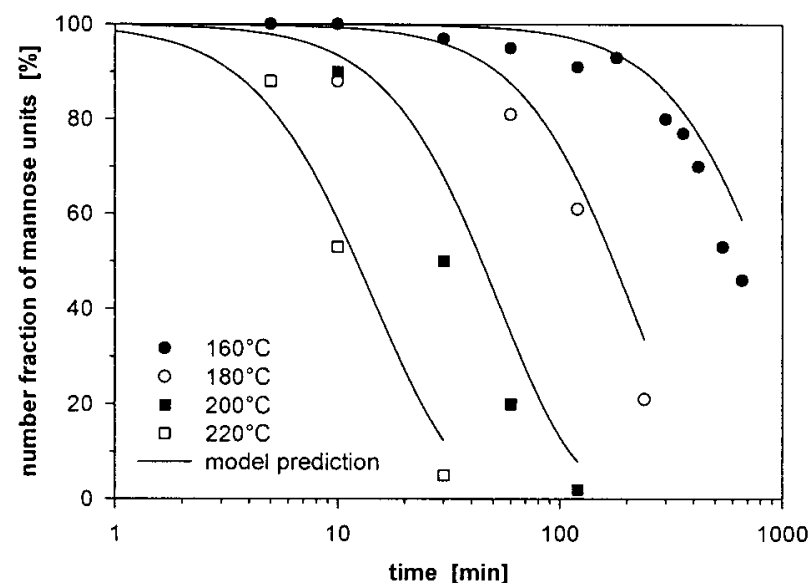

Figure 12. Prediction of the total mannose content. $T=160,180$, 200 , and $220^{\circ} \mathrm{C}$; rate constants from the fit of oligomer data.

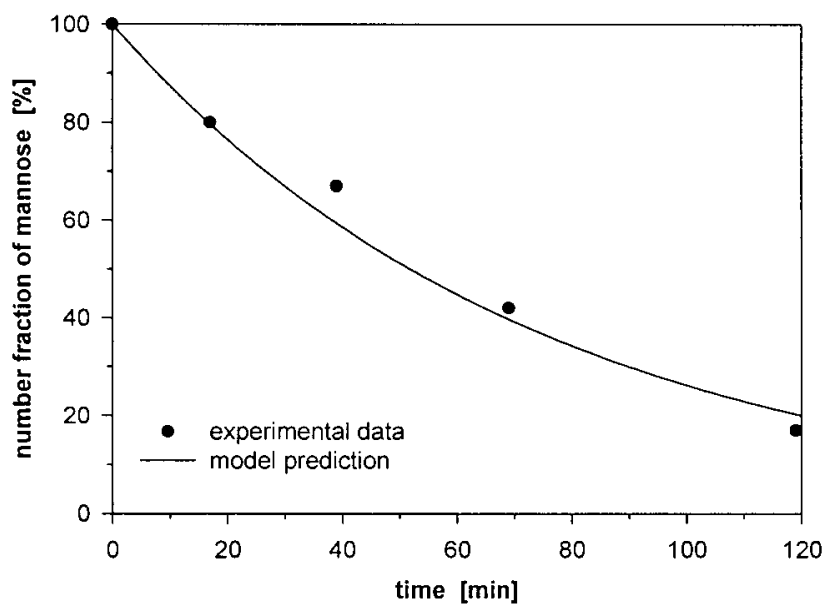

Figure 13. A priori prediction of mannose degradation. $T=180$ ${ }^{\circ} \mathrm{C}, \mathrm{K}_{\mathrm{D}}=0.0134 \mathrm{~min}^{-1}$ from the fit of mannan trials.

present results, but in such unbuffered trials the $\mathrm{pH}$ is unknown and can change during hydrolysis. It is hard to tell what degradation reactions are actually observed in an unbuffered system.

The model presented here is the simplest possible. First, the degradation rate is considered to be independent of the size of the molecule, which is confirmed by a trial performed with mannose at $180^{\circ} \mathrm{C}$, a case where only degradation can take place. The a priori prediction of the model (based on the $k_{D}$ value obtained from the mannan trials) fits the experimental data remarkably well (Figure 13). This is in contrast to the observation of a higher degradation rate for the monomer (kgtot $=$ $\left.0.45 \mathrm{~s}^{-1}\right)^{31}$ than for the dimer $\left(\mathrm{k} 1+\mathrm{k} 2=0.16 \mathrm{~s}^{-1}\right){ }^{13}$

Second, the hydrolysis rate in the present model is independent of the position of the bond and of the size of the fragment. This is contrary to theoretical arguments ${ }^{33}$ and experimental data on acid hydrolysis of xylo-ol igosaccharides ${ }^{14}$ and $\alpha$-(1,4)-glucans, ${ }^{15}$ which indicate that the glucosidic bond at the nonreducing end should be scissioned about twice as fast as the other bonds. This hypothesis was used in a Monte Carlo simulation of the production of glucose from amylose. ${ }^{17}$ However, a model with twice the hydrolysis rate at the nonreducing end does not fit our data. It predicts a much higher amount of monomer than what is observed (Figure 14). It could be that thermal hydrolysis as investigated in this work and acid hydrolysis as in those references are different in this respect.

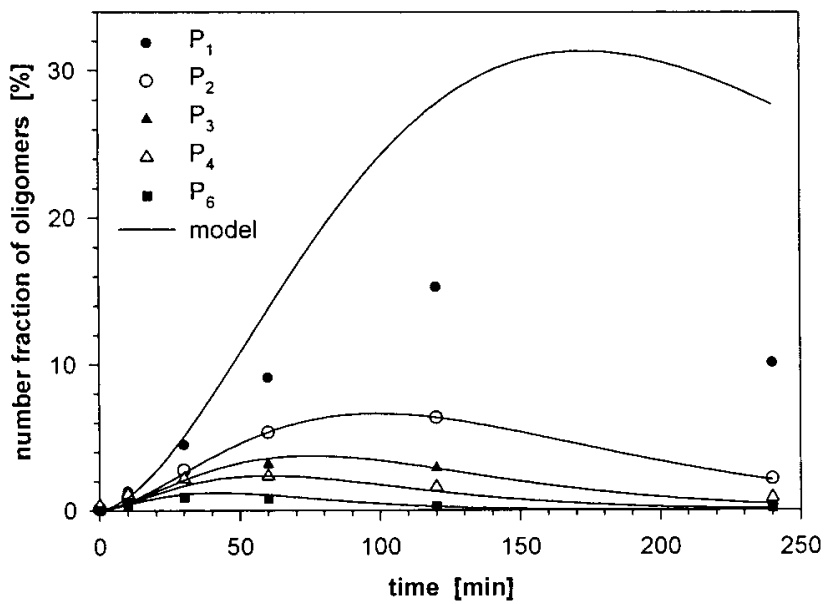

Figure 14. Rejection of the hypothesis of double reactivity at the nonreducing end. Comparison of experimental data with calculated curves, $\mathrm{T}=180^{\circ} \mathrm{C}$.

\section{Conclusion}

A mathematical model with random hydrolysis of glucosidic linkages and degradation of the reducing end was successfully applied to the thermal hydrolysis of mannan. The model has the advantage that production of small molecules is independent of the initial chainlength distribution as long as the average initial chain length is some 5 times higher than the largest species measured. Optimization of model parameters indicated that mannan thermal hydrolysis has an activation energy similar to those of recently published results for cellobiose. ${ }^{13}$ The activation energy for degradation is higher than that for hydrolysis, which may be related to the influence of the formate buffer. The present model can be applied to first-order depolymerization of other linear polymers with one link type in order to determine reaction rate constants or make predictions about molecular weight distribution on the basis of known reaction rate constants.

\section{Literature Cited}

(1) Bradbury, A. G. W.; Halliday, D. J . Chemical structures of green coffee bean polysaccharides. J . Agric. Food Chem. 1990, 38, 389.

(2) Monro, J . A.; Harding, W. R.; Russell, C. E. Dietary fibre of coconuts from a pacific atoll: soluble and insoluble components in relation to maturity. J . Sci. Food Agric. 1985, 36, 1013.

(3) Meier, H. On the structure of cell walls and cell wall mannans from ivory nuts and from dates. Biochim. Biophys. Acta 1958, 28, 229.

(4) Chanzy, H. D.; Grosrenaud, A.; Vuong, R.; Mackie, W. The crystalline polymorphism of mannan in plant cell walls and after recrystallisation. Planta 1984, 161, 320.

(5) Whistler, R. L. Solubility of polysaccharides and their behaviour in solution. Adv. Chem. Ser. 1973, 117, 242.

(6) Lüttge, U.; Kluge, M.; Bauer, G. Botanique; Tec et doc Lavoisier: Paris, 1992.

(7) Garves, K. Temperature, salt, and acidity effects on the hydrolysis of cellulose dissolved in concentrated acids. Cellulose Chem. Technol. 1996, 30, 3.

(8) Sasaki, M.; Kabyemela, B.; Adschiri, T.; Malaluan, R.; Hirose, S.; Takeda, N.; Arai, K. Cellulose hydrolysis in subcritical and supercritical water. J. Supercrit. Fluids 1998, 13, 261.

(9) Moiseev, Y. V.; Zaikov, G. E. Chemical Resistance of Polymers in Aggressive Media; Consultants Bureau: New York, 1987.

(10) Bobleter, O.; Bonn, G. The hydrothermolysis of cellobiose and its reaction product D-glucose. Carbohydr. Res. 1983, 124, 185.

(11) Coultate, T. P. Food, the Chemistry of its Components; The Royal Society of Chemistry: London, 1989. 
(12) Bunn, H. F.; Higgins, P. J . Reaction of monosaccharides with proteins: possible evolutionary significance. Science 1981, 213, 222.

(13) Kabyemela, B. M.; Takigawa, M.; Adschiri, T.; Malaluan, R. M.; Arai, K. Mechanism and kinetics of cellobiose decomposition in sub- and supercritical water. Ind. Eng. Chem. Res. 1998, 37, 357.

(14) Kamiyama, Y.; Sakai, Y. Rate of hydrolysis of xylo-oligosaccharides in dilute sulfuric acid. Carbohydr. Res. 1979, 73, 151.

(15) Weintraub, M. S.; French, D. Acid hydrolysis of $(1 \rightarrow 4)$ al pha-D-glucans. Carbohydr. Res. 1970, 15, 251.

(16) Basedow, A. M.; Ebert, K. H.; Ederer, H.J . Kinetic studies on the acid hydrolysis of dextran. Macromolecules 1978, 11, 774.

(17) Pinto, J . H. Q.; Kalaguine, S. A. Monte Carlo analysis of glucosidic bonds in polysaccharides. AIChE J . 1991, 37, 905.

(18) Kim, M. H.; Kim, C. S.; Lee, H. W.; Kim, K. Temperature dependence of dissociation constants for formic acid in aqueous solutions up to $175^{\circ} \mathrm{C}$. J . Chem. Soc., Faraday Trans. 1996, 92, 4951.

(19) Fried, J . R. Polymer Science and Technology; PrenticeHall: Englewood Cliffs, NJ , 1995.

(20) Ballauff, M.; Wolf, B. A. Degradation of chain molecules. 2. Thermodynamically induced shear degradation of dissolved polystyrene. Macromolecules 1984, 17, 209.

(21) Ballauff, M.; Wolf, B. A. Thermodynamically induced shear degradation. Advances in Polymer Science 85: Polymer Physics; Springer-Verlag: New York, 1988.

(22) J ellinek, H. H. G.; White, G. The degradation of long-chain molecules by ultrasonic waves. I. Theoretical. J . Polym. Sci. 1951, VI, 745.
(23) Mostafa, M. A. K. Degradation of addition polymers by ultrasonic waves. J. Polym. Sci. 1956, XXII, 535.

(24) Schaaf, E.; Zimmermann, H. Thermogravimetrische Untersuchung des thermischen und thermooxydativen Abbaus von Polyäthylenterephthalat. Faserforsch. Textiltech. 1974, 25, 434.

(25) Kuhn, W. Ueber die Kinetik des Abbaues hochmolekularer Ketten. Ber. Chem. Dtsch. Ges. 1930, 63, 1503.

(26) Guiata, M.; Chiantore, O.; Luda, M. P. Monte Carlo simulations of polymer degradations. 1. Degradations without volatilization. Macromolecules 1990, 23, 2087.

(27) Lowry, G. G. Markov Chains and MonteCarlo Calculations in Polymer Science; Marcel Dekker: New York, 1970.

(28) Ziff, R. M.; McGrady, E. D. Kinetics of polymer degradation. Macromolecules 1986, 19, 2513.

(29) Gear, C. W. Numerical I nitial ValueProblems in Ordinary Differential Equations; Prentice-Hall: Englewood Cliffs, NJ , 1971.

(30) Steiner, E.; Rey, T.; McCroskey, M. SimuSolv Reference Guide; Dow Co.: Midland, MI, 1990.

(31) Kabyemela, B. M.; Adschiri, T.; Malaluan, R. M.; Arai, K. Kinetics of glucose epimerization and decomposition in subcritical and supercritical water. Ind. Eng. Chem. Res. 1997, 36, 1552.

(32) Bobleter, O.; Pape, G. Der hydrothermale Abbau von Glucose. Monath. Chem. 1968, 99, 1560

(33) Feather, M. S.; Harris, J . F. The acid-catalysed hydrolysis of glycopyranosides. J . Org. Chem. 1965, 30, 153.

Received for review J anuary 13, 1999 Revised manuscript received April 26, 1999 Accepted May 9, 1999

IE 990034] 